\title{
Towards a Unified Classification of the Ectodermal Dysplasias: Opportunities Outweigh Challenges
}

\author{
Alan D. Irvine $1,2^{*}$ \\ 1Department of Clinical Medicine, Trinity College Dublin, Our Lady's Children's Hospital Crumlin, Dublin, \\ Ireland \\ 2Department of Paediatric Dermatology, Our Lady's Children's Hospital Crumlin, Dublin, Ireland
}

*Correspondence to:

Alan D. Irvine, M.D., Department of Clinical Medicine, Trinity College Dublin, Our Lady's Children's Hospital Crumlin, Dublin 12, Ireland.

E-mail: irvinea@tcd.ie

The ectodermal dysplasias include a complex and highly diverse group of heritable disorders that share in common developmental abnormalities of ectodermal derivatives. The broader definition of ectodermal dysplasias (as heritable disorders involving at least two of the ectodermal derivatives nails, teeth, hair, and eccrine sweat glands) encompasses 170-200 conditions. Some conditions included by this definition are relatively common; others are rare and, in some cases, family-specific. Classification of the ectodermal dysplasias has largely been approached by categorizing patterns of clinical findings (phenotypic grouping). In the last 2 decades great progress has been made in understanding the molecular pathogenesis and inter-relatedness of some of these conditions and a new consensus approach to classification that incorporates this new information is needed. A comprehensive and definitive classification of these disorders would be highly valuable for the many stakeholders in ED. As diseasespecific molecular treatments are developed, accurate classification will assume greater importance in designing registries to enable rapid identification of those with rare disorders who may wish to participate in clinical trials. Ideally a working classification of such a disparate collection of conditions would have a design and architecture that would facilitate easy accessibility by each of the key stakeholder groups and would encourage enhanced interaction between these parties. Attaining this objective is a major challenge but is achievable. This article reviews the historical-clinical perspective and the impact of recent developments in molecular biology in the field. Reflections are offered as to the future direction of classification systems in these disorders.

\section{INTRODUCTION}

The ectodermal dysplasias encompass a complex and highly diverse group of heritable disorders. By some authors' estimations, the broader definition of ectodermal dysplasias encompasses some 170-200 individual conditions. This article briefly discusses the historical-clinical perspective on defining and classifying these conditions and reviews the tremendous recent impact of developments in molecular biology. Harnessing these developments and reconciling them with clinical classifications offers rich promise if several obstacles can be overcome. Meeting this challenge will require input and collaboration from all the stakeholders involved.

\section{DEFINITIONS: WHAT TO INCLUDE AND EXCLUDE?}

Any approach to summarizing current knowledge about this group of diverse inherited conditions presents several challenges. Firstly and most importantly, how is an ectodermal dysplasia defined, and which distinct conditions are encompassed by this broad term? The history of the terminology throughout the literature is instructive. The first clinical cases with features of what would now be classified as ectodermal dysplasia were reported as early as 1792, when Danz [1792] reportedtwo Jewish boys with congenital absence of hair and teeth. Darwin, 1875 reported of a case ". . .communicated to me by Mr.Wedderburn, of a Hindoo family in Scinde inwhich 10 men, in the course of 4 generations, were furnished, in both jaws taken together, with only 4 small and weak incisor teeth and with 8 
posterior molars. The men thus affected have very little hair on the body, and become bald early in life. They also suffer much during hot weather from excessive dryness of the skin." This family would now be recognized as having X-linked hypohidrotic ectodermal dysplasia (HED) but the term "ectodermal dysplasia" did not appear until 1929 [Weech, 1929]. Prior to this report a small series of cases with hypotrichosis, hypodontia, onychodysplasia, and anhidrosis had been described under various names such as "dystrophy of hair and nails", "imperfect development of skin, hair, and teeth", and "congenital ectodermal defect". The designation outlined by Weech specified three essential aspects of ectodermal dysplasias: (1) Most of the disturbances must affect tissues of ectodermal origin; (2) These disturbances must be developmental; (3) Heredity plays a causal role. Weech had in mind the X-linked anhidrotic form (Christ-Siemens-Touraine syndrome (CST) or HED, OMIM: 305100) in males but noted that it had also been reported in females; he also noted that this pattern of involvement was occasionally inherited as a non-sexlinked trait. For some authors and clinicians, the term ectodermal dysplasia is still used specifically with reference to CST syndrome and the autosomal dominant and recessive forms of HED. As more clinical reports of patients with similar but subtly distinct patterns of anomalies were recorded, the term "ectodermal dysplasia" became extended to include many different genetic entities. In an attempt to encapsulate this heterogeneity and diversity of symptoms seen, Touraine [1936] suggested the expression "ectodermal polydysplasia". Attempts at more formal classification soon followed; initially conditions were classified as hidrotic or anhidrotic, but this simple classification failed to reflect the complexity of nail, hair, and dental anomalies associatedwith the various forms of ED.

Currently the most widely accepted and used definition of the ectodermal dysplasia is of a group of inherited disorders that share in common developmental abnormalities of two or more of the following: hair, teeth, nails, sweat glands, and other ectodermal structures. Other structures derived from embryonic ectoderm include the mammary gland, thyroid gland, thymus, anterior pituitary, adrenal medulla, central nervous system, external ear, melanocytes, cornea, conjunctiva, lacrimal gland, and lacrimal duct. There are merits and demerits of this approach to definition. One definite benefit is that the problems encountered by many patients and families are similar regardless of the specific subtype of ED; parents and children can benefit by being part of larger support networks exemplified by the Ectodermal Dysplasia Society (UK-based; URL: www.ectodermaldysplasia.org) and the National Foundation for Ectodermal Dysplasias (US-based; URL: http:// www.nfed.org). This wide-ranging classification is also helpful in concentrating the minds of research workers in the field and several EDs are now known to have shared genetic mechanisms. While the broader definition has the benefit of reach and inclusivity, many conditions encompassed by this broad definition are often not usually considered as primarily ectodermal dysplasias. For example, inherited conditions as diverse as incontentia pigmenti, dyskeratosis congenita, trichothiodystrophies, cardiofaciocutaneous syndrome, pachyonychia congenita and Goltz syndrome are all included by this definition as ectodermal dysplasias but common practice has been to consider many of these as separate entities. In excess of 190 different conditions are encompassed under the umbrella term of "ectodermal dysplasia", if the widest used definition of "an inherited disorder involving two or more of the following ectodermal appendages: teeth, hair, and eccrine sweat glands" is used, for a recent detailed summary of most of these conditions Irvine [2005]. Thus, before a definitive classification effort can proceed, agreement needs to be reached as to which inclusion and exclusion criteria are to applied to identify a definitive set of conditions to be included.

\section{CLASSIFICATION: CLINICAL APPROACHES}

Having accepted the broadest definition of an ectodermal dysplasia, the second challenge presented by this group of conditions is that of designing a meaningful and functional classification system. Until the end of the 20th century, classification systems for ectodermal dysplasias were, due to lack of molecular understanding, based on clinical manifestations. Several authors addressed the issue of delineating nosologic groups of conditions linked by shared phenotypic traits. The most comprehensive accounts of clinical phenotypes and inheritance patterns of ectodermal dysplasia were produced by Freire-Maia and Pinheiro [1984] in their classic monograph and in subsequent writings [Pinheiro and Freire-Maia, 1994]. Their classification designated conditions by groups depending on presence of hair, nail, tooth, or sweat gland abnormalities, and assigned conditions to groups using a "1-2-3-4 system" to collate conditions that had involvement of hair (1), teeth (2), nails (3), or sweat glands (4) to groups such as 12-n or 123-n. This classification was a comprehensive, indeed magisterial, attempt at ordering an unwieldy group of conditions but was not intuitive to use and grouped together disparate clinical entities such as Goltz 
syndrome and pachyonychia congenita. In common with any other classification of ectodermal dysplasias based on clinicalfindings this system is confounded by the subtleties of inheritance such as incomplete penetrance and variable expressivity of phenotype. This is especially true in the ectodermal dysplasia where sweating is often not formally measured and tooth or nail anomalies may be subtle. For a comprehensive contemporaneous consideration of the breadth of ED conditions in the tradition of FriereMaia and Pinheiro [Irvine, 2005].Nowclinical classifications need to become more focussed, more userfriendly, and better integrated with emerging molecular data. Difficult decisions will need to be made to balance inclusiveness (of every possible ectodermal dysplasia) with accessibility and practicality of usage.

\section{CLASSIFICATION: MOLECULAR APPROACHES}

The last decade has seen several important insights into the molecular basis of several of the ectodermal dysplasias. In some cases the molecular data have confirmed clinical impressions, for example Hay-Wells syndrome and ectrodactyly, ectodermal dysplasia, clefting (EEC) syndrome have ectodermal dysplasia and clefting of the palate and lip as common clinical findings and these conditions are now known to be allelic [McGrath et al., 2001]. Prompted by the great advances in molecular knowledge several authors have proposed new molecular-based approached [Priolo and Lagana, 2001; Lamartine, 2003; Itin and Fistarol, 2004]. These proposed approaches classify conditions based on the class of molecule responsible for the disorder, for example categorizing together those with mutations in structural or developmental molecules. This approach has many advantages, especially for characterization of defects in preparation for molecular diagnostics and, hopefully, molecular therapy. These systems, however, need to be integrated with clinical findings and need to be accessible to all clinicians involved in the care of these patients. Importantly, it has to be realized that currently it is not possible to provide a molecular diagnosis for all patients, even in those with classical clinical abnormalities of conditions well characterized at a molecular level. Molecular factors such as unusual mutation mechanisms can frustrate conventional mutation identification as can health care service delivery factors such as access to and affordability of molecular diagnostics; these and other confounders are likely to remain the case in the foreseeable future. If a molecular-based system is to function well for lesscommon conditions, it will need to be supported by the generation of contemporary and regularly updated online databases such as the intermediate filament database (www.interfil.org) that will allow physicians and researchers to share data and to access detailed genotype/phenotype correlation.

\section{THE WAY FORWARD: TOWARDS AN INTEGRATED, ACCESSIBLE, AND CONTEMPORARY CLASSIFICATION SYSTEM}

Once decisions are taken on inclusion and exclusion criteria, any new classification system will have to allow incorporation of all the current clinical and molecular knowledge and "build in" sufficient flexibility to adjust and revise when new data emerge. Following on this necessity for flexibility lies the greatest challenge of all: the need to provide a system or, as seems more likely, integrated systems (i.e., clinical, molecular, needs-based) that speak to each stakeholder in the process: the family with an affected childwho wish to learn more about their condition and meet with others experiencing similar issuesregardless of the precise molecular diagnosis, the physician or health professional struggling with a difficult diagnosis or the treatment of a rare condition, the genetic counselor explaining inheritance patterns and recurrence risks, the research scientist examining basic molecular mechanisms or, as may be increasingly likely, designing specific molecular therapy for a rare condition. As more mechanistic data are gleaned about ectodermal dysplasias, disorder- or even mutation-specific treatments are likely to emerge. When these treatments become available, accurate classification will be essential if wellcharacterized patients are to be placed in registries waiting clinical therapeutic trials. Thus, the prize of a functional, integrated system for classifying ectodermal dysplasias is great and is truly worth a concerted effort by all those involved. Current molecular advances and the enthusiasm of so many in the field, in concert with the strong encouragement of the NFED mean that now is the time to take up this challenge.

\section{ACKNOWLEDGMENTS}


The author wishes to acknowledge the support of the NFED in preparing this work. This paper was presented at the International Conference on Ectodermal Dysplasia, Charleston, South Carolina, March $10-12,2008$. 


\section{REFERENCES}

Danz D. 1792. Sechste Bemerkung. Von Menschen ohne Haare und Zahne. Stark Arch Geburth Frauen Neugeb Kinderkr 4:684.

Darwin C. 1875. The variations of animals and plants under domestication. Vol. 2.2nd edition. London: John Murray. p 319.

Freire-Maia N, Pinheiro M. 1984. Ectodermal dysplasias: A clinical and genetic study. New York: Alan R. Liss.

Irvine AD. 2005. Ectodermal dysplasias. In: Harper JI, Oranje OP, Prose N, editors. Pediatric dermatology. Oxford: Blackwell Scientific. pp 1412-1466.

Itin PH, Fistarol SK. 2004. Ectodermal dysplasias. Am J Med Genet Part C 131C:45-51.

Lamartine J. 2003. Towards a new classification of ectodermal dysplasias. Clin Exp Dermatol 28:351-355.

McGrath JA, Duijf P, Doetsch V, Irvine AD, de Waal R, Vanmolkot K, Wessagowit VA, Kelly K, Atherton DJ, Griffiths WAD, Orlow SJ, van Haeringen A, Ausems M, Yang A, McKeonF, BamshadMA, Brunner HG, Hamel BCJ, van Bokhoven H. 2001.

Hay-Wells syndrome is caused by heterozygous missense mutations in the SAM domain of p63. Hum Mol Genet 10:221-229.

Pinheiro M, Freire-Maia N. 1994. Ectodermal dysplasias: A clinical classification and a causal review. Am J Med Genet 53:153-162.

Priolo M, Lagana C. 2001. Ectodermal dysplasias: A new clinical-genetic classification. J Med Genet 38:579-585.

Touraine A. 1936. L'anidrose h_er_editaire avec hypotrichose et anodontie (polydysplasie ectodermique h_er_editaire). Presse M_ed 44:145-149.

Weech A. 1929. Hereditary ectodermal dysplasia (congenital ectodermal defect). A report of two cases. Am J Dis Child 37:766-790. 